\title{
Financement des PME au Québec : portrait comparatif
}

\author{
Adalbert Nkoudou Bessala ${ }^{1}$ \\ LEST-CNRS UMR 6123 - Aix-Marseille Université, France
}

\section{INTRODUCTION}

Dans son Enquête sur le financement et la croissance des petites et moyennes entreprises réalisée par Industrie Canada et Statistique Canada en $2014^{2}$, cinq types de financement externes ont été examinés : l'emprunt, le crédit- bail, le crédit commercial, le capital-actions et le financement gouvernemental. Cette enquête va également inclure le rapport de l'entrepreneur au financement personnel lors du lancement d'une nouvelle entreprise.

\section{PART DES PME DANS L'ÉCONOMIE : DÉFINITION ET CADRAGE STATISTIQUE}

\subsection{Définition : approche statistique de la PME}

L'approche statistique de la PME se fonde sur deux variables fondamentales pour définir la petite et moyenne entreprise : le chiffre d'affaires et le nombre d'employés. Ainsi, pour Statistique Canada peut être considérée comme petite et moyenne entreprise toute organisation qui compte de 1 à 499 employés inclusivement et dont le chiffre d'affaires ne dépasse pas 50 millions de dollars et n'est pas inférieur à 30000 dollars. Ce type d'entreprise peut être subdivisé en deux sous-catégories : les petites entreprises qui comptent de 1 à 99 employés inclusivement, et les moyennes entreprises qui emploient entre 100 et 499 salariés inclusivement.

\subsection{Cadrage statistique du nombre pondéré de PME en 2014}

Que l'on soit au Québec ou ailleurs au Canada, la proportion en nombre pondéré de PME est en croissance. En 2014, le Canada comptait environ 621147 petites et moyennes entreprises en nombre pondéré. La majorité des PME sont de petites entreprises (environ 98,4\%), c'est-à-dire des organisations comptant moins de 100 employés. Ce constat est valable que l'on soit au Québec ou au Canada.

Tableau 1 - Répartition des PME au Canada en 2014 selon l'effectif

\begin{tabular}{|c|c|r|r|}
\cline { 3 - 4 } \multicolumn{2}{c|}{} & Nombre pondéré & \multicolumn{1}{l|}{$\%$} \\
\hline Toutes les PME & 1 à 499 employés & 621147 & 100,0 \\
\hline \multirow{2}{*}{ Effectif } & 1 à 99 employés & 611389 & 98,4 \\
\cline { 2 - 4 } & 100 à 499 employés & 9758 & 1,6 \\
\hline
\end{tabular}

Source : Statistique Canada, Enquête sur le financement et la croissance des petites et moyennes entreprises, 2014.

\section{L'analyse des données nous permet de mesurer le poids économique des PME selon les différentes régions et territoires du Canada.}

Autres faits saillants relativement au rôle stratégique des PME dans l'économie : au Québec en 2014, environ 40 PME de 1 à 499 employés sur 100 sont localisées dans la région de Montréal $(41,96 \%)$. Cette proportion est supérieure de 32,67 points de pourcentage à celle observée dans la ville de Québec. À l'échelle du Canada, 10 PME de 1 à 499 employés sur 100 sont localisées à Montréal, contre 1 PME sur 100 dans la ville de Québec. Au regard de ces données, nous pouvons dire que Montréal reste le cœur économique du Québec. De même, l'ancrage territorial des PME au Québec est proportionnel à la répartition de la population active du Québec. L'analyse des données nous permet de mesurer le poids économique des PME selon les différentes régions et territoires du Canada. En effet, $21,7 \%$ de ces 
PME se trouvaient au Québec en 2014, contre $36,1 \%$ en Ontario, 19,3\% dans les Prairies, $7,1 \%$ dans l'Atlantique; et enfin $15,8 \%$ en ColombieBritannique et les territoires. Au regard de ces données, nous pouvons conclure que la localisation géographique des PME au Canada est proportionnelle à la répartition globale de la population active, la majorité des PME étant installée en Ontario (36,1 \%) et au Québec $(21,7 \%)$.

Le fait que la majorité des PME soit installée sur la partie d'un territoire densément peuplé ne doit pas être considéré comme acquis. Du point de vue de l'action publique, elle pose la question du «nomadisme et de l'ancrage territorial » des petites et moyennes entreprises (Zimmermann, 1998) ${ }^{3}$. Autrement dit, comment les acteurs publics, censés soutenir la création et le développement, peuvent-ils favoriser sa pérennité à l'échelle locale?

Le tableau ci-dessous regroupe la répartition des PME par zone géographique au Canada en 2014.

Tableau 2 - Nombre pondéré par région des PME en 2014 au Canada

\begin{tabular}{|c|c|c|c|}
\hline & & Nombre pondéré & $\%$ \\
\hline \multirow{12}{*}{ Région } & Atlantique & 43837 & 7,1 \\
\hline & Québec & 134788 & 21,7 \\
\hline & Ville de Québec & 12519 & 2,00 \\
\hline & Montréal & 56551 & 9,10 \\
\hline & Ontario & 224234 & 36,1 \\
\hline & Nord de l'Ontario & 15919 & 2,6 \\
\hline & Sud de l'Ontario & 208315 & 33,5 \\
\hline & Prairies & 119973 & 19,3 \\
\hline & Manitoba & 18804 & 3,0 \\
\hline & Saskatchewan & 20381 & 3,3 \\
\hline & Alberta & 80788 & 13,0 \\
\hline & Colombie-Britannique et les territoires & 98315 & 15,8 \\
\hline
\end{tabular}

Source : Statistique Canada, Enquête sur le financement et la croissance des petites et moyennes entreprises, 2014.

Avec un taux de 98,4\% des PME existantes au Canada en 2014, nous pouvons prétendre que les petites entreprises qui regroupent des entreprises qui emploient de 1 à 99 salariés jouent un rôle stratégique dans l'économie. Par conséquent, nous estimons qu'il est important de comprendre leurs besoins et les processus d'accès au financement dans le but de favoriser leur croissance et leur compétitivité.

\section{FINANCEMENT EXTERNE : PROFIL DES PME QUI ONT FAIT UNE DEMANDE}

\section{La proportion des PME qui ont fait une demande de financement externe a tendance à augmenter avec leur taille et leur âge.}

Dans cette section, nous présenterons une brève analyse des informations recueillies sur les taux de demande de financement des PME. Ces données nous permettront de faire une brève analyse des facteurs qui augmentent la probabilité qu'un entrepreneur au Québec fasse une demande de financement externe.

La taille, l'âge et la région géographique ont une influence sur la demande de financement externe des petites et moyennes entreprises.
Au Québec comme au Canada, la proportion des PME qui ont fait une demande de financement externe a tendance à augmenter avec leur taille et leur âge. En 2014, environ 46 PME de 1 à 499 employés sur 100 au Québec $(45,7 \%)$ et au Canada $(51,3 \%)$ ont fait une demande de financement externe. Cette proportion est supérieure de 8,5 points de pourcentage pour les petites entreprises qui comptent de 1 à 4 salariés inclussivement. Dans le même temps, cette proportion est inférieure de 19,9 points pour les moyennes entreprises $(71,2 \%)$, c'est-à-dire les organisations qui comptent de 100 à 499 employés.

Dans ce document, nous qualifions de «jeune $\mathrm{PME}$ », le fait d'être une entreprise en activité 
depuis 2 ans ou moins, et d'avoir un chiffre d'affaires qui est compris entre 30000 dollars et inférieur ou égal à 50 millions de dollars. Ainsi en 2014, plus de 5 PME sur 10 en activité depuis 2 ans ou moins ont manifesté plus de besoins de financement externe au Canada. Cette proportion est plus faible de 7,2 points de pourcentage pour les «PME anciennes ». Cette dernière catégorie regroupe toutes les entreprises qui sont âgées de 20 ans ou plus, qui emploient de 1 à 499 employés et ont un chiffre d'affaires compris entre 30000 dollars et 50 millions de dollars.

Par ailleurs, en 2014, le fait d'être une jeune PME encourage les entrepreneurs à solliciter du financement externe. À l'analyse des données ci-dessous, nous pouvons prétendre que la probabilité de solliciter du financement externe s'accroît avec l'âge de la PME.

Tableau 3

\begin{tabular}{|c|l|r|r|}
\cline { 3 - 4 } \multicolumn{2}{c|}{} & $\begin{array}{c}\text { Financement } \\
\text { externe demandé } \\
\text { en 2014 en \% }\end{array}$ & $\begin{array}{c}\text { N'a pas demandé de } \\
\text { financement externe en } \\
\text { 2014 en \% }\end{array}$ \\
\hline \multirow{3}{*}{$\begin{array}{c}\text { Âge de } \\
\text { l'entreprise }\end{array}$} & 2012 à 2014 (2 ans ou moins) & 58,0 & 42,0 \\
\cline { 2 - 4 } & 2004 à 2011 (3 à 10 ans) & 51,3 & 48,7 \\
\cline { 2 - 4 } & 1994 à 2003 (11 à 20 ans) & 49,8 & 50,2 \\
\cline { 2 - 4 } & Avant 1994 (plus de 20 ans) & 50,8 & 49,2 \\
\hline
\end{tabular}

Source : Statistique Canada, Enquête sur le financement et la croissance des petites et moyennes entreprises, 2014.

C'est au Québec que le taux de demande de financement externe des PME $(45,7 \%)$ ayant de 1 à 499 employés est le plus bas. Pour les entreprises de même taille au Manitoba, ce taux est le plus élevé avec $64,2 \%$ alors que la moyenne canadienne se situe à $51,3 \%$.
En 2014, dans la région de Toronto, plus de 5 PME sur 10 de 1 à 499 employés ont sollicité un financement externe. Dans le cas de Montréal, la proportion est inférieure pour les entreprises de taille identique (4 PME sur 10).

Tableau 4 - Taux de demande de financement externe des PME en 2014 selon la région du Canada

\begin{tabular}{|c|c|c|c|c|}
\hline & & & \multirow[b]{2}{*}{$\begin{array}{l}\text { Financement } \\
\text { externe } \\
\text { demandé en } \\
2014 \text { en \% }\end{array}$} & \multirow[b]{2}{*}{$\begin{array}{l}\text { N'a pas demandé de } \\
\text { financement externe } \\
\text { en } 2014 \text { en \% }\end{array}$} \\
\hline & & & & \\
\hline \multirow{13}{*}{ Région } & \multicolumn{2}{|l|}{ Atlantique } & 49,6 & 50,4 \\
\hline & \multicolumn{2}{|l|}{ Québec } & 45,7 & 54,3 \\
\hline & & Ville de Québec & 43,5 & 56,5 \\
\hline & & Montréal & 44,0 & 56,0 \\
\hline & \multicolumn{2}{|l|}{\begin{tabular}{|l|} 
Ontario \\
\end{tabular}} & 54,8 & 45,2 \\
\hline & & \begin{tabular}{|l|} 
Toronto \\
\end{tabular} & 51,6 & 48,4 \\
\hline & & Nord de l'Ontario & 62,6 & 37,4 \\
\hline & & Sud de l'Ontario & 54,2 & 45,8 \\
\hline & \multicolumn{2}{|l|}{\begin{tabular}{|l|} 
Prairies \\
\end{tabular}} & 54,8 & 45,2 \\
\hline & & Manitoba & 64,2 & 35,8 \\
\hline & & Saskatchewan & 58,6 & 41,4 \\
\hline & & Alberta & 51,7 & 48,3 \\
\hline & $\begin{array}{l}\text { Colombie-Britannique } \\
\text { et les territoires }\end{array}$ & & 47,3 & 52,7 \\
\hline
\end{tabular}

Source : Statistique Canada, Enquête sur le financement et la croissance des petites et moyennes entreprises, 2014. 
À l'analyse des données ci-dessus nous pouvons conclure que la probabilité qu'une «jeune PME » ou une «PME ancienne» fasse une demande de financement externe s'accroît avec la taille, l'âge de l'entreprise et sa localisation géographique. Ceci nous semble valable au Québec et au Canada.

\section{SOURCES ET APPROBATION DES DEMANDES DE CAPITAUX : QUI FINANCE L'ENTREPRENEURIAT ET LES PME AU QUÉBEC}

\section{Dans l'ensemble, que l'on soit au Québec ou au Canada, nous notons un très faible recours des PME au financement offert par une institution gouvernementale.}

Dans cette section, nous aborderons tout d'abord, la problématique des sources de financement de l'entrepreneur au moment du démarrage d'une PME. Ensuite, nous présenterons le taux d'approbation du financement par emprunt.

Dans cette communication, nous qualifions de financement par emprunt l'ensemble des programmes de financement offerts par une banque à charte canadienne, une coopérative de crédit/caisse populaire et enfin par une institution gouvernementale. Ce financement par emprunt peut être accordé sous forme de prêt à terme, d'hypothèque non résidentielle, de marge de crédit et/ou de carte de crédit.

Pour financer le lancement de leur PME, en 2014, plus de 7 entrepreneurs sur $10(78,6 \%)$ au Québec, et plus de 8 entrepreneurs sur $10(84,3 \%)$ au Canada ont utilisé leurs épargnes personnelles. Cette source de financement a été également la plus souvent utilisée pour financer le lancement d'une entreprise à Montréal ( 8 entrepreneurs sur 10, soit $82,9 \%)$. Cette proportion est plus élevée de 3,2 points de pourcentage à Toronto $(86,1 \%)$.

En 2014, d'après l'enquête d'Industrie Canada et de Statistique Canada, plus de 4 PME sur 10 $(47,9 \%)$ au Québec ont bénéficié d'un financement d'une banque à charte canadienne. Cette proportion est essentiellement identique à celles qui ont reçu un financement d'une coopérative de crédit/caisse populaire $(47,1 \%)$. Par contre, au Québec, à peine une 1 PME sur $10 \mathrm{a}$ obtenu un financement d'une institution gouvernementale. Au Canada, le scénario est différent, alors que plus de 7 PME sur $10(71,6 \%)$ ont eu recours au financement d'une banque à charte canadienne.
Soulignons qu'en Ontario cette proportion est très élevée avec plus de 8 PME sur 10 (89,8 \%). D'ailleurs, dans l'ensemble du Canada, le taux de recours au financement d'une coopérative de crédit/caisse populaire $(24,7 \%)$ est plus faible de 22,4 points de pourcentage par rapport au Québec.

Autre fait saillant à Montréal, métropole économique du Québec, le taux de recours au financement d'une banque à charte canadienne (65\%) est de $17,1 \%$ plus élevé que celui de l'ensemble du Québec. Cette proportion est largement plus faible de 26,3 points de pourcentage dans la région de Québec $(38,7 \%)$.

Dans l'ensemble, que l'on soit au Québec ou au Canada, nous notons un très faible recours des PME au financement offert par une institution gouvernementale, soit $8,4 \%$ pour le Québec et $6,2 \%$ au Canada. Cependant, soulignons que la proportion de financement gouvernemental obtenu par une PME est très élevée en Saskatchewan $(17 \%)$ et dépasse les proportions canadienne et québécoise respectivement de 10,8 et 8,6 points de pourcentage.

En résumé, en 2014, les PME québécoises ont eu un accès insuffisant au financement offert par une institution gouvernementale.

$\mathrm{Au}$ regard des données précédentes se pose maintenant la question suivante : quel montant global les PME du Québec ont-elles reçu en matière de financement par emprunt en 2014 ?

Au Québec, le montant total autorisé du financement par emprunt en 2014 s'élevait à 7,8 milliards de dollars pour un montant total demandé de 8,5milliards de dollars, soit un ratio montant total autorisé/demandé de $91,7 \%$. Au Canada, le ratio montant total demandé (32,5 milliards de dollars) versus montant total autorisé $(28,02$ milliards de dollars) est plus faible, soit $86,1 \%$. 
En somme, en 2014, les principales sources de financement au moment du démarrage d'une PME au Québec sont les économies personnelles et les prêts contractés auprès de ses amis ou des membres de sa famille. Cet aspect du financement de la création d'entreprises en phase de démarrage est également valide dans le reste du Canada.

Par contre, en phase de croissance et de développement, les PME du Québec ont davantage recours au financement par emprunt offert par les banques à charte canadienne $(47,9 \%)$ et les coopératives de crédit/caisses populaires $(47,1 \%)$. Au Canada, le scénario est différent. La principale source de financement externe des PME est celle offerte par les banques à charte canadienne. Encore une fois, peu importe que l'on soit au Québec ou au Canada, la proportion des PME qui ont fait une demande de financement auprès d'une institution gouvernementale est très faible, voire marginale ou nulle (comme à Saint John avec $0 \%$ ).

À la lecture des données présentées plus haut, nous pouvons expliquer le faible recours aux financements offert par les institutions financières gouvernementales par deux phénomènes. Tout d'abord, nous pouvons prétendre que le très faible recours au financement des institutions gouvernementales au Québec et au Canada est la preuve qu'il existe des effets de trappe en ce qui concerne l'accès au financement des institutions gouvernementales censées soutenir la création de PME en phase de démarrage et de croissance (Nkoudou, 2012) ${ }^{4}$. Ici, l'effet de trappe se manifeste par le fait que l'entrepreneur est incapable de rédiger son plan d'affaires selon la grille établie par les institutions gouvernementales. Cette incapacité peut découler de la difficulté de l'entrepreneur à expliciter son savoir-faire et son savoir d'action tant à l'oral qu'à l'écrit (Nkoudou, 2016) ${ }^{5}$

Ensuite, dans son rapport sur les aides publiques aux entreprises, Levet et al., (2003) ${ }^{6}$ soulignent que les aides publiques à la création et à la croissance des entreprises peuvent être source «d'effet d'aubaine». En effet, les aides ne déclenchent jamais l'intention de l'entrepreneur d'investir, de lancer une nouvelle entreprise, d'embaucher ou d'innover. Dans ce cas, la PME aurait investi ou embauché avec ou sans financement public (Nkoudou, 2012).

\section{BIBLIOGRAPHIE ET NOTES}

${ }^{1}$ Docteur en Sciences de gestion. Thèmes de recherche: Entrepreneuriat, innovation, PME, transfert et gestion des connaissances, efficacité et limites des politiques publiques d'innovation sur le territoire, GRH.

${ }^{2}$ Source: Statistique Canada (2014). Enquête sur le financement et la croissance des petites et moyennes entreprises.

${ }^{3}$ Zimmermann, J. B. (1998). Nomadisme et ancrage territorial: propositions méthodologiques pour l'analyse des relations firmes - territoires. Revue d'économie régionale et urbaine, 1998-2.

${ }^{4}$ Nkoudou Bessala, A. (2012) Statut et gestion des savoirs du créateur-dirigeant dans l'innovation de produits en petite et moyenne entreprise. Implication en matière de politique publique sur le territoire (Thèse de doctorat inédite). Aix-Marseille Université, LEST/CNRS.

${ }^{5}$ Nkoudou Bessala, A. (2016, juin). Gestion des savoir-faire et savoir d'action au sein des PME technologiquement innovantes: proposition d'un cadre conceptuel. Communication présentée à la IX ${ }^{\mathrm{e}}$ conférence de l'Agecso European Business School, à Paris, France.

${ }^{6}$ Levet J.-L., Astie Griffith, C., Gavini, C., Guellec J., Maisonneuve A. et Namur, D. (2003). Les aides publiques aux entreprises: une gouvernance, une stratégie. Dans J-L. Levet (dir.), Rapport (197 p.). Paris, France : La Documentation française. 


\section{FORMULAIRE D'ABONNEMENT}

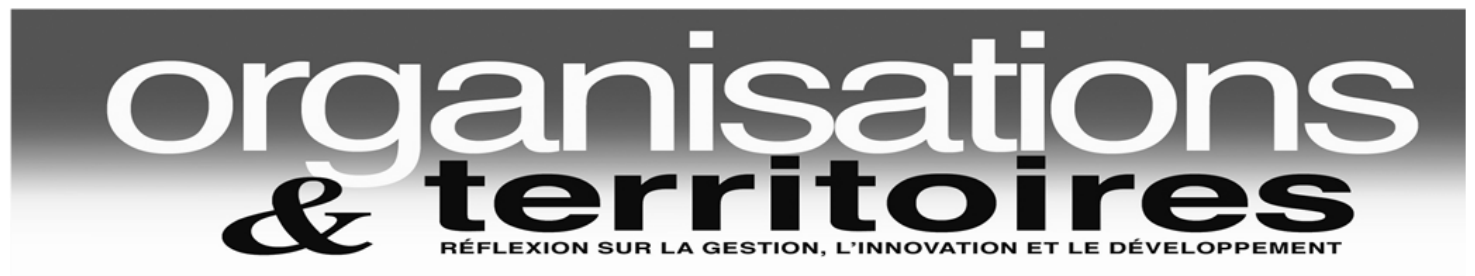

Je désire m'abonner à la revue Organisations et territoires et je joins un chèque au montant de : $\$$

Nouvel abonné $(\sqrt{ }): \square \quad$ Renouvellement $(\sqrt{ }): \square \quad$ Date :

Abonnement institutionnel (6 numéros) 103,48 \$ (Toutes taxes incluses)

Abonnement individuel (6 numéros) 66,69 \$ (Toutes taxes incluses)

Abonnement étudiant (6 numéros) 48,29 \$ (Tx inc.) (Envoyer photocopie carte étud.)

Abonnement hors Canada (6 numéros) $145,00 \$ \quad(110 \$+35 \$$ frais de poste) (sans taxes)

Tous les abonnements sont d'une durée de deux ans

- J'aimerais que mon abonnement débute avec le DERNIER numéro paru, soit :

Volume $\mathrm{N}^{\circ}$

- J'aimerais que mon abonnement débute avec le PROCHAIN numéro à paraître, soit :

Volume $\mathrm{N}^{\circ}$

Faites-nous parvenir vos coordonnées :

Nom :

Organisation :

Adresse :

Téléphone :

Télécopieur :

Courriel :

Adresse d'expédition : $\quad$ UQAC - Revue Organisations et territoires 555, boulevard de l'Université, bureau H6-1360

Chicoutimi (Québec) CANADA G7H 2B1

Téléphone : (418) 545-5011, poste 4530 Télécopieur : (418) 693-9072

Courriel : revueot@uqac.ca Site Web : www.uqac.ca/revueot 